\title{
Reinventing Technology and Innovation Management using a Three Dimensional approach
}

This paper was downloaded from TechRxiv (https://www.techrxiv.org).

\section{LICENSE}

CC BY 4.0

\section{SUBMISSION DATE / POSTED DATE}

05-01-2021 / 10-01-2021

\section{CITATION}

Gopalakrishnan, Abhilash (2021): Reinventing Technology and Innovation Management using a Three Dimensional approach. TechRxiv. Preprint. https://doi.org/10.36227/techrxiv.13524026.v1

$\mathrm{DOI}$ 


\title{
Reinventing Technology and Innovation Management using a Three Dimensional approach
}

\author{
Abhilash Gopalakrishnan \\ Senior Member, IEEE \\ E-mail: abhilash.g.in@ieee.org
}

\begin{abstract}
In recent years, we are observing turbulence across sectors of the economy, geography, technology or business. This turbulence manifests itself in multiple forms like megatrends, natural disasters and pandemics. The lifetimes of organizations reduced to twenty four years by 2016 and expected to be twelve years by 2027. We can see these changes across three dimensions of change as trend storms, rate of change and disruption. By identifying best practices across successful organizations we propose a three-dimensional canvas and approach of applying agility and leadership to deal with rate of change, trend analysis to take advantage of them and identifying and nurturing change-makers in the organization. Agility and the winning characteristics of inspirational leadership and operating like a network plays a pivotal role. Dissecting the maker revolution we set the stage for identifying and nurturing makers internally within the organization. We knit these elements together in the organizational fabric by using customer journey maps, data, and road-mapping. Integrating these, we propose a canvas and approach enabling one step towards continuous innovation. These tools will enable leaders and organizations to better integrating emerging technologies, trends and innovation into their execution strategies.
\end{abstract}

Index Terms-Product, Systems, Trends, Industry 4.0, Disruption, Makers, Customer Journeys, Agile.

\section{INTRODUCTION}

In recent years, we are observing turbulence across sectors of the economy, geography, technology or business. This turbulence manifests itself in multiple forms like mega trends, natural disasters, and pandemics. Half a century ago life expectancy for Fortune 500 organizations was around seventy five years. This meant that the need for re-invention was a once in a lifetime affair. Fast-forward to today. The lifetimes of organizations reduced to twenty-four years by 2016 and expected to be twelve years by 2027 . This would mean one out of three companies would cease to exist in their current form over the next five years. [14].

For many product and services organizations developing and maintaining great products and services for business comes with the traditional challenges including trade-offs, dynamics, details, time pressure, and economics [1]. Combined with these, the current trend storms are coming with radical changes induced by Digital and Industry 4.0. Industry 4.0 symbolizes smart systems. The technology universe is massive considering the technologies of Artificial Intelligence (AI), 3D Printing, Robotics, Internet of Things(IoT), Autonomous Vehicles, nanotechnology, biotechnology, materials, energy storage, and quantum computing to name a few. It is this fusion of these technologies and their interaction across physical, digital, and biological domains that make the fourth industrial revolution different from previous revolutions. The revolution is nothing less than a storm because of the velocity, the breadth, depth, and impact on systems and society. These leverage the pervasive power of digitalization and information technology, with data at its heart [2]. We observe that most organizations are not prepared for the change in breadth and depth and often confusing these with short-term gains.

In our observation, incumbent organizations comfortable with their business model are pushed out of their comfort zones. Stealth mode startups following lean methods are outsmarting these incumbents with their radical approaches to technology and business. They can pivot or change forms faster while the bigger organizations struggle and often take many years to react, within which there emerges a major gap. The difference-maker is often seen as preparation [3].

In the confusion, many organizations are seen creating new divisions and filling these with new people. The internal capabilities are often ignored [4]. We believe this is a capability building exercise and might need a balance between internal abilities and external ones extending to partners. Therefore we observe many an organization, as well as individual, is at crossroads. The challenge for leadership is to take a meaningful course of action considering the impact across trend storms, rate of change and, disruption while integrating it with the organizational landscape. While technology can be an enabler for organizing in various forms and functions, we believe these have a multi-dimensional effect. The challenges across a three dimensional landscape can be summarized as in Fig. 1.

Peter Drucker's statement once said 'What we need is an entrepreneurial society in which innovation and entrepreneurship are normal, steady and continuous'. Peter often envisaged a vehicle for the future with the customer in the driving seat, with the environment in view through dashboard and mirrors, riding on the wheels of knowledge, innovation, people, and collaboration. As individuals, industry and decision-makers, we all have this big question, are we going to be disrupted? The purpose of this paper is to present a canvas and approach in the direction of the vehicle for the future.

\section{The Approach Across Three Dimensions}

Like Steve Jobs once said 'We need to start with the customer experience and work backwards towards technol- 


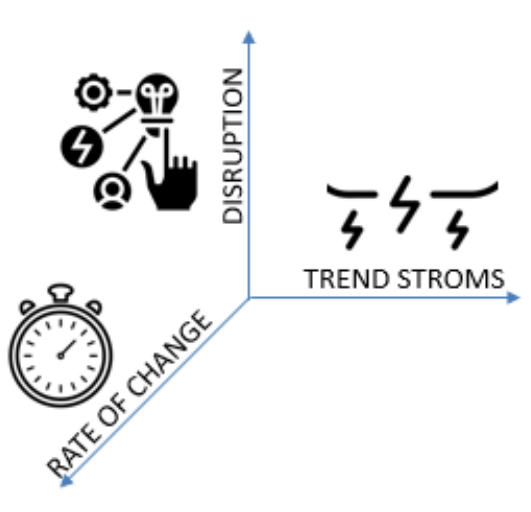

Fig. 1: Challenges across three dimensions

ogy'. For instance, Cloud lets the data collected processed elsewhere, Internet of Things lets us reach every nook and corner unraveling new data, while machine learning lets us identify the patterns from data and enables insights through analytics. Artificial Intelligence enables us to perceive the environment and achieve goals. While these are enablers, what matters is the resulting customer value. The framework looks at the challenge across the three dimensions of trend storms, rate of change, and disruption. It proposes a combination of agility, trend analysis, and drive action through initiating more of making. We also discuss integrating these in the organizational execution landscape. To address the rate of change we see an approach of agility and leadership would help. To address technology storms we propose an approach using trend analysis and insight generation while dealing with disruptions by creating a work culture of change with support from change agents.

\section{A. Agility and Inspirational Leadership as the answer to the rate of change}

It is often mentioned that the best way to predict the future is to invent it. Similarly, by embracing change we can improve our existing solutions. . In the context of agile a growth mindset, which is an attitude to be open to change is a prerequisite. Let us observe the Cheetah, the fastest animal which can accelerate from zero to 60 meter per hour in three seconds. It is often identified it is not the speed rather the ability to leap sideways, changing direction, slowing down quickly which enables Cheetah to be effective. Let us now shift focus to the poster boys of agile like Spotify or Netflix or on the Systems Engineering side, Tesla or SpaceX. It is quite evident they have similar abilities to flock around the customer, organized as a network with small teams [5]. These structural and behavioral traits are the key behind agility which are represented in Fig. 2. With agility, it is proven that we can build a culture of continuous change which can enable us to deal with change. It is important also to notice the fundamental questions remain the same, are my customers happy? Are we building the right thing?

When we mean agility requires a network-like approach, it

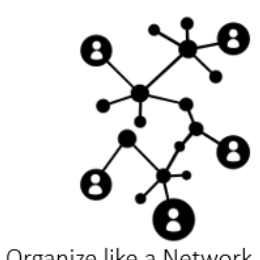

Organize like a Network

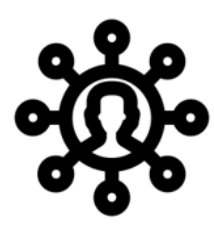

Customer Focus

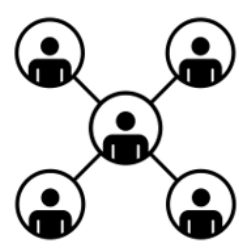

Small Team
Fig. 2: Agile Approach

is clear it is an integration of top-down and bottom-up that can lead to such a network. Leadership has a key role to play. An agile leader is seen to employ a pull approach which is inspirational in nature by supporting learning and tolerance to failure. We see great examples in leaders like Satya Nadella, the CEO of Microsoft saying, 'learn it all does it better than the know it all'. Richard Branson talks about failure as a reality and trusting people to learn from their mistakes. It is often observed that speed is often the most visible outcome of being agile while the others are mostly ignored. For instance, when E. Sreedharan, an Indian Railways Engineer, and his team performed the reconstruction of the pampan bridge, what was reported was the completion in forty-six days compared to six months target set by railways. It is therefore important to note that the thinking of Sreedharan in integrating the resources and people across railway workshops, fishermen in identifying girders on the seabed and teaming up with experts in lifting large equipment led to speed as an outcome [6]. In short, agile can be seen as the best effort to adapt to changes. At its heart of the integration is the consideration of value, data, and team.

\section{B. Trend Analysis as the answer to technology storms}

To address the information explosion we need to place an approach of turning data into insights. Sherlock Holmes, the fictitious character created by Sir Arther Conon Doyle is attributed to democratizing this approach often called 'Ladder of Inference'. This is the whole concept of observation, continuously being curious, being observing and bringing your observations, and selecting the data points in priority. And affixing meaning straight, creating assumptions conclude leading to conclusions, beliefs, and actions. And then again, this whole system again feeding back to the pool of observable data, thus improving the inference logic is what many leaders use as their insights. This is part of the leadership exercise as well. When in an agile landscape, every individual can be wearing a leadership hat, the need for this approach to be more organized and accessible to everyone is important. The approach is represented in Fig. 3.

Its possible to build on the hypothesis based on initial analysis integrating industry reports we have other reports, like the technology reports from IEEE, MIT Technology Review, of reports from McKinsey or Gartner or ARC Review to name a few. These information can be further used to do a tech mining [7]. The existence of patents in the area could also indicate a strategic interest. We need to curate this whole 


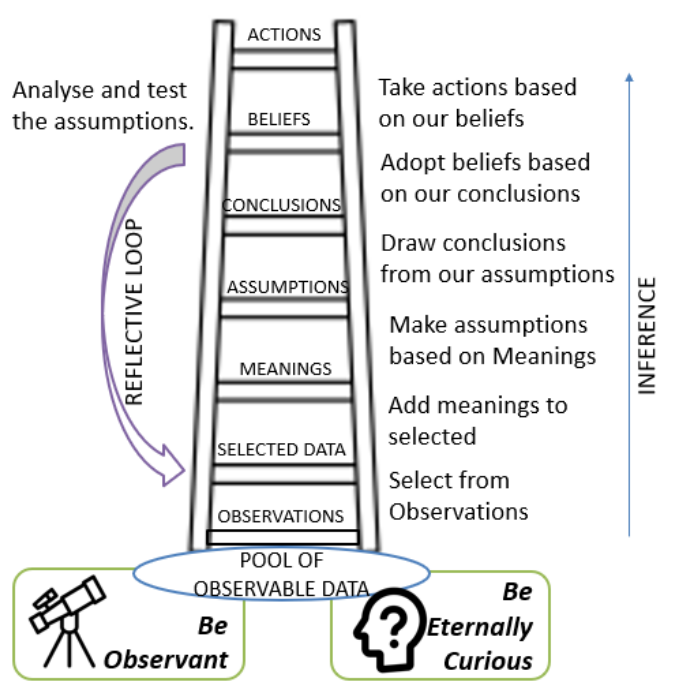

Fig. 3: Trend Analysis

information, prioritize it, elevate it. Typical industry analysis reports today form a Mutually Exclusive and Collectively Exhaustive (MECE) approach immortalized by McKinsey. These trends need to be posted in their organizational trend landscape in a kind of sunshine chart [8]. This leads to affixing a meaning for each in our short-term, medium-term and long-term context. The trend and its maturity level can be identified. NASA Technology Maturity Level is a decision factor to place it in the short and medium landscape [9]. Most of the leaders we know have developed their unique ways of mentally analyzing the data and deriving insights, which can very well be also supplemented with technology to keep pace with the rate of change. Thriving in Chaos through continuous reinvention is a major emerging theme.

The biggest question remains! How do we get it into execution? The elements of the approach need to be correlated and integrate into the overall execution strategy leading to actions. We need to make the best of the maker revolution, which is driven by makers. Chris Andersen refers to this as 'when the web generation turns to the real world' [16]. 3D printing kind of symbolizes the concept of industrial capability in the hands of everyone. This enables people who can use digital tools, at the comfort of their homes, Do It Yourself (DIY), to share these designs by open source and other online methods following standards. The open standards allow them to send designs to commercial manufacture and produce it in big numbers. There is a pressing need to identify and nurture change-makers within every organization. Competitions like hackathons, pilots, and lighthouse projects are suggested approaches to take ideas from initial concept to industry [10].

We can just look at Silicon Valley and the culture a little closer we see a fertile combination of academia, research, and collaboration. This is represented by Stanford University, Apple's Cupertino labs, Google's labs, HP, Oracle, Tesla. Stanford Dean of Engineering Frederick Terman's advice of

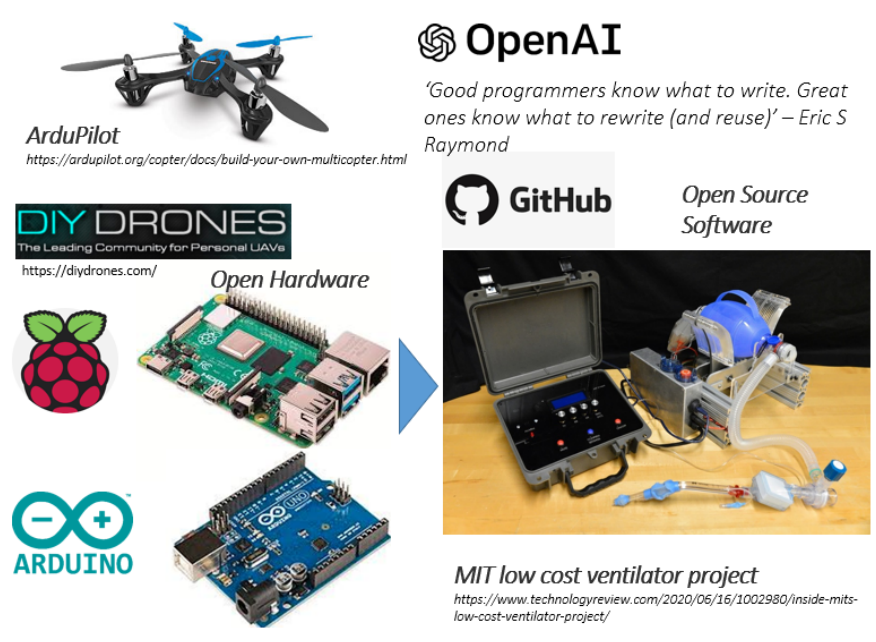

Fig. 4: Maker Revolution

staying in the area and build a hi-tech region was what inspired HP founders Bill Hewlett and David Packard to build HP in the area. The Homebrew Computer Club was an early computer hobbyist group in Menlo Park, the members included the likes of Steve Jobs and Steve Wozniak. This represents a making culture, which is at the heart of Silicon Valley. They also have the changing mindset and capability to disrupt themselves which allowed them to survive over many years.

Today, we have also a lot of open hardware designs available like Arduino, Raspberry Pi, DIY drones, open AI, and open source software. These elements of the world-wide tinkering revolution seems to be taking more societal value forms, like in MIT low-cost ventilator pilot. Like how Michael Scrage mentions in his book 'Serious Play' about simulations as continuous experimentation in bigger organizations, magic comes out of academia, industry, as communities, integrated by enthusiastic makers who embrace change.

Embracing the mega revolution would mean all these aspects to come together. We need a three-dimensional ecosystem, which is balanced across trends, agility well balanced between art science and technology. Moreover, this machine of research and innovation should work together to benefit humanity.

The approach positions ourselves in the wake of the storms, by placing ourselves to take advantage of the changes, by staying in its eye. Integrating these into the organizational operating system is important. This would mean we need an organized way of integrating these and channel the efforts. This is where approaches like the Three Layer Product Model suggested by Jan Bosch is highly beneficial [17]. With our understanding of the domain, we can organize the trends in a 3LPM diagram as commodity layer elements, differentiating functionality elements, and experimentation functionality elements. Layers visualization being one widely understood across varied stakeholders, it provides immense value as a visualization approach. Some trends like cloud are having impact across multiple areas. Some trends like digital twins for 


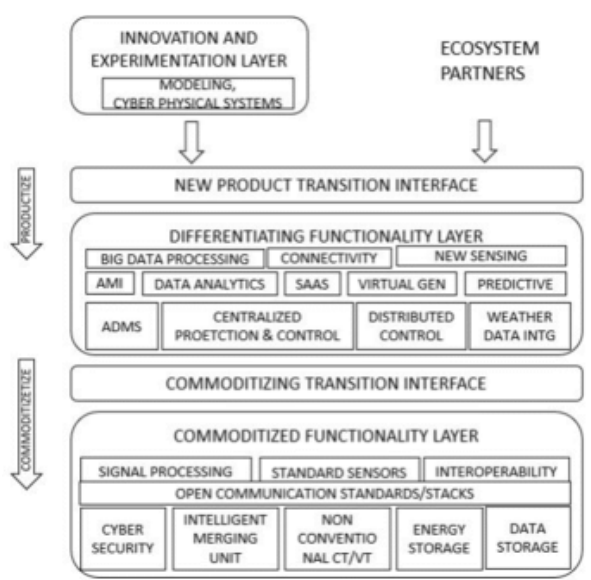

Fig. 5: 3 Layer Product Model Example for Power Systems

example fit well in an experimentation layer. Some aspects like machine learning might be relevant in differentiation layer 5 . This is a continuous process of landscaping and updated as the industry evolves. This visualization helps us build or reorganize road-maps thus changing directions to suit better positioning in the industry market. This visualization also helps us think and reorganize our people based on skills and competencies.

We need to integrate value-driven engineering is important to be part of the approach [19]. This is done by building a user journey driven by empathy to identify the pains and gains. In the earlier dimension of product development, we were considerably happy with the way we were talking about stakeholders in their roles like tester, engineer, field engineer, operator. Today, when companies are flocking around customers, it is important to notice focus has shifted from delighting the customer more than just satisfying them. There is a need for a deeper understanding and perspective of each individual as a stakeholder in terms of their pains and gains to identify opportunities based on latent needs. An approach based on design thinking leading to customer journey mapping and working with the customer to identify pains and gains is important here. Approaches to co-create with customers and arriving at early prototypes enabling experimentation is an important element which is accelerated also by cloud and software-based approaches.

Another key aspect to run our part of the organization in a lean startup mode, basing on data to arrive at decisions [20]. We need to look at operational data that is one thing which we are kind of lacking me sometimes most of our organizations are thinking we don't have that is the kind of talk that goes on. But in lean-agile startup, we are talking about a continuous improvement process where we should look at measuring the effectiveness of lean, and learn by analyzing data. Earlier we were employing an approach of feedback loop by user interviews and discussions. Today, if we can crossover to continuous deployment, we can augment these with data which allows us to stay closer to the customer. This ability can

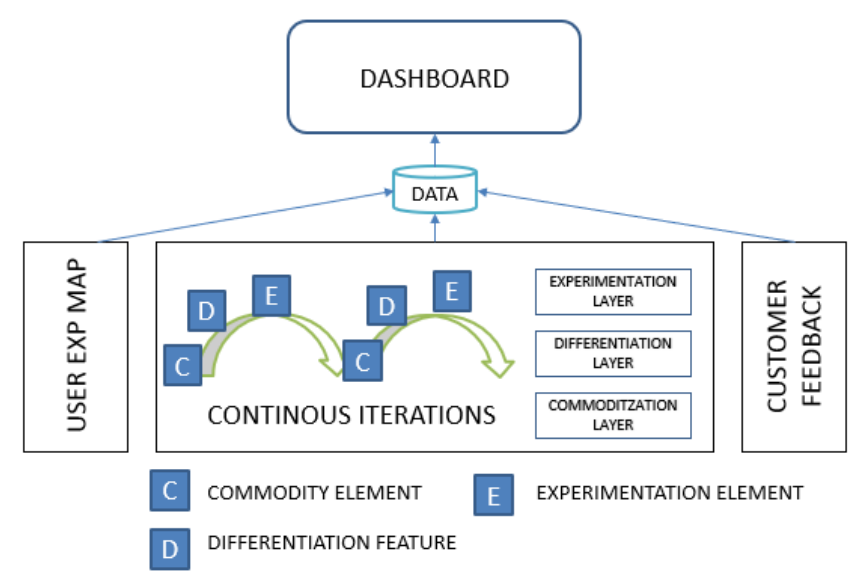

Fig. 6: Overall approach

further enable us to move from perceived business value to real business value and improving the insights in the journey.

\section{CONCLUSION}

We discussed the key challenges and summarized them across three dimensions of change as trend storms, rate of change and disruption. Building on the vehicle of future approach, by identifying best practices across successful organizations we propose a three-dimensional canvas and approach of applying agility and leadership to deal with the rate of change, trend analysis to take advantage of them and identifying, and nurturing change-makers within the organization. Agility and the winning characteristics of inspirational leadership and operating like a network plays a pivotal role. Dissecting the maker revolution we set the stage for identifying and nurturing makers internally within organization. As we noticed that the borders between strategy and execution blurring in a lean movement, we knit these elements together in the organizational fabric by using customer journey maps, data, and road-mapping. This enables innovation to be part and parcel of organizational execution thus moving one step towards continuous innovation. The dashboard is considered to evolve into a navigation and guidance system for the organization. We believe these tools will enable leaders and organizations to better integrating emerging technologies, trends and innovation into their execution strategies.

\section{REFERENCES}

[1] Karl T. Ulrich, Steven D. Eppinger, "Product Design and Development" 5th edition, ISBN-13: 978-0073404776, 2012.

[2] Klaus Schwab, “The Fourth Industrial Revolution”, Jan 2017.

[3] Walter Frick, 'How to Survive a Recession and Thrive Afterward'. Harward Business Review ,2019.

[4] Wade Burgess, 'Why Companies Overlook Great Internal Candidates,, Harvard Business Review, 2016.

[5] Stephen Denning, "The Age of Agile: How Smart Companies are Transforming the Way Work Gets Done", March 2018.

[6] Ashokan M S, Rajesh Rajamohan, 'Karmayogi: A Biography of E. Sreedharan'. 
[7] Alan L. Porter,Scott W. Cunningham, 'Tech Mining: Exploiting New Technologies for Competitive Advantage', 2005. [8] Cambridge Roadmapping
https://www.cambridgeroadmapping.net/templates

templates:

[9] Alison Olechowski, Steven D. Eppinger, Nitin Joglekar,'Technology Readiness Levels at 40: a study of state-of-the-art use, challenges, and opportunities, MIT Open access arctiles, 2015.

[10] World Economic Forum, 'Lighthouses for a perfect storm', Dec 2019.

[11] Samuel Greengard, 'The Internet of Things", MIT Press, 2015.

[12] Nayan B. Ruparelia, 'Cloud Computing”, MIT Press, 2016.

[13] Elizabeth Haas Edersheim, 'The Definitive Drucker: Challenges For Tomorrow's Executives - Final Advice From the Father of Modern Management', 2007

[14] Nadya Zhexembayeva, 'The Chief Reinvention Officer Handbook: How to Thrive in Chaos', Nov 2020

[15] Clayton M. Christensen , 'The Innovator's Dilemma: When New Technologies Cause Great Firms to Fail', 2016.

[16] Chris Anderson, René Ruiz, et al., 'Makers: The New Industrial Revolution', 2012.

[17] Jan Bosch, 'Achieving Simplicity with the Three-Layer Product Model', IEEE Computer.

[18] Yuxun Zhou, Reza Arghandeh et. al., 'Big Data Application in Power Systems', Elsevier, 2017.

[19] Schmarzo, B. 'The Economics of Data, Analytics, and Digital Transformation: The theorems, laws, and empowerments to guide your organization's digital transformation', Packt, 2020.

[20] Eric Ries, 'The Lean Startup: How Today's Entrepreneurs Use Continuous Innovation to Create Radically Successful Businesses', 2011. 